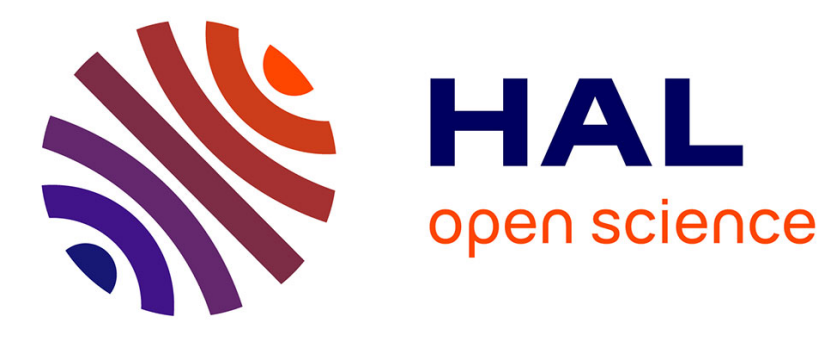

\title{
The crystal structure of K2FeF5
}

\author{
Marcus Vlasse, G. Matejka, Alain Tressaud, B. M. Wanklyn
}

\section{To cite this version:}

Marcus Vlasse, G. Matejka, Alain Tressaud, B. M. Wanklyn. The crystal structure of K2FeF5. Acta crystallographica Section B: Structural crystallography and crystal chemistry, 1977, 33 (11), pp.33773380. 10.1107/S0567740877011030 . hal-00156618

\section{HAL Id: hal-00156618 https://hal.science/hal-00156618}

Submitted on 21 Jun 2007

HAL is a multi-disciplinary open access archive for the deposit and dissemination of scientific research documents, whether they are published or not. The documents may come from teaching and research institutions in France or abroad, or from public or private research centers.
L'archive ouverte pluridisciplinaire HAL, est destinée au dépôt et à la diffusion de documents scientifiques de niveau recherche, publiés ou non, émanant des établissements d'enseignement et de recherche français ou étrangers, des laboratoires publics ou privés. 
Acta Crystallographica Section B

Structural

Science

ISSN 0108-7681

Editor: Carolyn P. Brock

\section{The crystal structure of $\mathrm{K}_{2} \mathrm{FeF}_{5}$}

M. Vlasse, G. Matejka, A. Tressaud and B. M. Wanklyn

Copyright $($ International Union of Crystallography

Author(s) of this paper may load this reprint on their own web site provided that this cover page is retained. Republication of this article or its storage in electronic databases or the like is not permitted without prior permission in writing from the IUCr. 
Acta Cryst. (1977). B33, 3377-3380

\title{
The Crystal Structure of $\mathrm{K}_{2} \mathrm{FeF}_{5}$
}

\author{
By M. Vlasse, G. Matejka and A. Tressaud \\ Laboratoire de Chimie du Solide du CNRS, Université de Bordeaux I, 351 cours de la Libération, \\ 33405 Talence CEDEX, France
}

AND B. M. WANKLYN

Clarendon Laboratory, University of Oxford, Oxford, England

(Received 17 March 1977; accepted 22 April 1977)

\begin{abstract}
The structure of $\mathrm{K}_{2} \mathrm{FeF}_{5}$ has been refined for 1088 counter intensities to $R=0.048$. It is orthorhombic, space group $P n 2{ }_{1} a$, with $a=20.39$ (1), $b=7.399$ (5), $c=12.84$ (1) $\AA, Z=16$. The structure is made up of infinite zigzag cis chains of $\mathrm{FeF}_{6}$ octahedra with common corners running along a. One-dimensional magnetism may be predicted for this compound.
\end{abstract}

\section{Introduction}

The fluorides of transition metals are of interest because of their physical properties and the diversity of their structures. Anderson (1963) and Goodenough (1963) showed that the magnetic couplings in these fluorides are primarily hy superexchange which facilitates the magnetic study and the establishment of relationships between structure and properties.

The study of fluorides with a general formula $A_{x} M_{y} \mathrm{~F}_{z}$, where $A$ is a monovalent cation and $M$ a $3 d$ transition element, and in particular of the systems $A \mathrm{~F}-$ $\mathrm{FeF}_{3}-\mathrm{FeF}_{2}(A=\mathrm{Li}, \mathrm{Na}, \mathrm{K}, \mathrm{Rb}, \mathrm{Tl})$ has allowed the derivation of a structural classification explaining their magnetic properties (Portier, Tressaud, de Pape \& Hagenmuller, 1968; Tressaud, Portier, de Pape \& Hagenmuller, 1970). In this classification the phases $A \mathrm{FeF}_{3}$ have three-dimensional octahedral lattices, the phases $A_{3} \mathrm{Fe}_{2} \mathrm{~F}_{7}$ are composed of double layers of octahedra, the phases $A \mathrm{FeF}_{4}, A_{2} \mathrm{FeF}_{4}$ and $A_{5} \mathrm{Fe}_{3} \mathrm{~F}_{14}$ are made up of single octahedral sheets, and the phases $A_{2} \mathrm{FeF}_{5}$ have octahedral chain structures. The study of $\mathrm{Na}_{5} \mathrm{Fe}_{3} \mathrm{~F}_{14}$ has brought about a partial verification of this classification (Vlasse, Menil, Moriliere, Dance, Tressaud \& Portier, 1976; Tressaud, Dance, Vlasse \& Portier, 1976). The structural determination of $\mathrm{K}_{2} \mathrm{FeF}_{5}$ will further check this systematic description and allow a better explanation of the magnetic properties.

\section{Experimental}

\section{Specimen preparation}

Single crystals were prepared by the flux method in a fluorinating atmosphere. The synthesis was carried out in a $\mathrm{Pt}$ crucible containing $\mathrm{PbCl}_{2}, \mathrm{KF}$ and $\mathrm{FeF}_{3}$. This mixture was raised to $650^{\circ} \mathrm{C}$ and then cooled to $330^{\circ} \mathrm{C}$ at the rate of $1.5^{\circ} \mathrm{C} \mathrm{h}^{-1}$. Colourless transparent crystals were obtained with a maximum size of $4 \times$ $3 \times 3 \mathrm{~mm}$. Their powder pattern was similar to those of $\mathrm{K}_{2} \mathrm{CrF}_{5}$ and $\mathrm{K}_{2} \mathrm{GaF}_{5}$ (Wanklyn, 1975).

Table 1. Atomic coordinates $\left(\times 10^{4}\right)$ and isotropic temperature factors for $\mathrm{K}_{2} \mathrm{FeF}_{5}$, with standard deviations in parentheses

\begin{tabular}{|c|c|c|c|c|}
\hline & $x$ & $y$ & $z$ & $B\left(\AA^{2}\right)$ \\
\hline $\mathrm{Fe}(1)$ & $4224(3)$ & $34(15)$ & $2505(6)$ & $0.42(5)$ \\
\hline $\mathrm{Fe}(2)$ & $3294(3)$ & $6744(6)$ & $521(6)$ & $0.79(6)$ \\
\hline $\mathrm{Fe}(3)$ & $1721(4)$ & 0 & $544(6)$ & $0.78(6)$ \\
\hline $\mathrm{Fe}(4)$ & $783(4)$ & $6661(16)$ & $2534(7)$ & $1.27(7)$ \\
\hline $\mathrm{K}(1)$ & $43(5)$ & $8483(18)$ & $176(9)$ & $1.56(9)$ \\
\hline $\mathrm{K}(2)$ & $1662(4)$ & $4732(13)$ & $420(7)$ & $1.11(7)$ \\
\hline$K(3)$ & $850(4)$ & $1703(15)$ & $2791(6)$ & $0.99(6)$ \\
\hline $\mathrm{K}(4)$ & $2511(6)$ & $3453(17)$ & 3224 (3) & $0.84(7)$ \\
\hline $\mathrm{K}(5)$ & $4181(5)$ & 4880 (17) & $2632(7)$ & $1.51(8)$ \\
\hline$K(6)$ & $2483(9)$ & $8325(30)$ & 2877 (4) & $1.76(9)$ \\
\hline$K(7)$ & $4914(4)$ & 8240 (17) & $187(8)$ & $0.96(8)$ \\
\hline $\mathrm{K}(8)$ & 3394 (5) & $1785(15)$ & $339(8)$ & $1.72(9)$ \\
\hline$F(1)$ & $2211(10)$ & $256(30)$ & $4636(15)$ & $1.42(17)$ \\
\hline$F(2)$ & $2015(13)$ & $1273(39)$ & $1787(21)$ & $2 \cdot 39(24)$ \\
\hline$F(3)$ & 4600 (12) & $1180(35)$ & $1331(20)$ & $0.50(20)$ \\
\hline$F(4)$ & 3494 (14) & $1652(39)$ & $2349(20)$ & $3.89(30)$ \\
\hline$F(5)$ & $4721(9)$ & $1560(31)$ & $3378(15)$ & $0.77(13)$ \\
\hline$F(6)$ & $977(9)$ & 1555 (14) & 484 (16) & $1.62(17)$ \\
\hline$F(7)$ & 1379 (14) & $3160(40)$ & $4453(25)$ & $2.50(29)$ \\
\hline$F(8)$ & 3584 (11) & $3340(32)$ & $4507(20)$ & $1.34(17)$ \\
\hline$F(9)$ & $4041(12)$ & $5440(39)$ & $488(23)$ & 2.84 (14) \\
\hline$F(10)$ & $1521(10)$ & $5263(28)$ & $2463(17)$ & 0.34 (14) \\
\hline$F(11)$ & $322(13)$ & $5147(22)$ & $3442(24)$ & $2.81(30)$ \\
\hline$F(12)$ & $416(15)$ & $5657(41)$ & $1263(24)$ & $3.29(31)$ \\
\hline$F(13)$ & $2784(10)$ & $6191(29)$ & 4491 (15) & $1.07(16)$ \\
\hline$F(14)$ & $2444(8)$ & $8157(24)$ & $721(8)$ & 0.90 (12) \\
\hline$F(15)$ & 4989 (10) & $8343(34)$ & $2388(15)$ & $0.97(11)$ \\
\hline$F(16)$ & $3721(4)$ & $8397(44)$ & $1581(23)$ & $3.25(31)$ \\
\hline$F(17)$ & $1210(9)$ & $8436(28)$ & $1519(15)$ & 0.41 (13) \\
\hline$F(18)$ & 3882 (14) & $8819(38)$ & $3623(21)$ & $2.55(28)$ \\
\hline$F(19)$ & 1104 (12) & 8241 (35) & 3577 (18) & $1.44(21)$ \\
\hline$F(20)$ & 2959 (14) & $5403(41)$ & $1598(23)$ & $1.58(26)$ \\
\hline
\end{tabular}




\section{Single-crystal diffraction data}

Weissenberg and precession photographs indicate orthorhombic Laue symmetry $\mathrm{mmm}$. The systematic extinctions, $0 k l: k+l=2 n$ and $h k 0: h=2 n$, are consistent with space groups $P n 2, a$ and Pnma. The lattice parameters refined from single-crystal data are $a=20.39$ (1), $b=7.399$ (5), $c=12.84$ (1) $\AA$. The cell contains 16 formula units $\left[d_{x}=3 \cdot 16(2), d_{c}=3 \cdot 14 \mathrm{~g}\right.$ $\mathrm{cm}^{-3}$.

A single crystal in the form of a rectangular block
$0.07 \times 0.09 \times 0.16 \mathrm{~mm}$ was mounted about $\mathbf{b}$. The intensities were collected on an Enraf-Nonius CD-3 three-circle automatic diffractometer with Mo $K a$ radiation $(\lambda=0.70942 \AA)$, a pyrolytic graphite monochromator (002) and a $4^{\circ}$ take-off angle. A scintillation counter and a $\theta-2 \theta$ multiple scanning technique with a scan rate of $10^{\circ}(2 \theta) \mathrm{min}^{-1}$ were used. The background was taken at each end of the scan range for a time equal to the actual scan time. Three control reflexions measured for every batch of 50 reflexions showed a random fluctuation of about $6 \%$.

Table 2. Interatomic distances (in $\AA \pm 0.009 \AA$ ) and bond angles $\left[\text { in }\left(^{\circ}\right) \pm 0.1^{\circ}\right]_{\text {for }} \mathrm{K}_{2} \mathrm{FeF}_{5}$

$\begin{array}{ll}\mathrm{Fe}(1)-\mathrm{F}(3) & 1.892 \\ \mathrm{Fe}(1)-\mathrm{F}(4) & 1.921 \\ \mathrm{Fe}(1)-\mathrm{F}(5) & 1.886 \\ \mathrm{Fe}(1)-\mathrm{F}(15 a) & 2.005 \\ \mathrm{Fe}(1)-\mathrm{F}(16 a) & 1.982 \\ \mathrm{Fe}(1)-\mathrm{F}(18 a) & 1.832 \\ \langle\mathrm{Fe}(1)-\mathrm{F}\rangle & 1.920 \\ & \\ \mathrm{~K}(1)-\mathrm{F}(5 e) & 3.008 \\ \mathrm{~K}(1)-\mathrm{F}(5 b) & 2.757 \\ \mathrm{~K}(1)-\mathrm{F}(6 f) & 2.991 \\ \mathrm{~K}(1)-\mathrm{F}(6 g) & 2.662 \\ \mathrm{~K}(1)-\mathrm{F}(8 b) & 2.931 \\ \mathrm{~K}(1)-\mathrm{F}(12) & 2.624 \\ \mathrm{~K}(1)-\mathrm{F}(12 g) & 2.625 \\ \mathrm{~K}(1)-\mathrm{F}(15 d) & 3.128 \\ \mathrm{~K}(1)-\mathrm{F}(17) & 2.937 \\ \mathrm{~K}(1)-\mathrm{F}(18 d) & 2.834 \\ \langle\mathrm{~K}(1)-\mathrm{F}\rangle & 2.850 \\ & \\ \mathrm{~K}(5)-\mathrm{F}(4) & 2.793 \\ \mathrm{~K}(5)-\mathrm{F}(5) & 2.857 \\ \mathrm{~K}(5)-\mathrm{F}(8) & 2.929 \\ \mathrm{~K}(5)-\mathrm{F}(9) & 2.799 \\ \mathrm{~K}(5)-\mathrm{F}(11 j) & 2.712 \\ \mathrm{~K}(5)-\mathrm{F}(12 j) & 2.947 \\ \mathrm{~K}(5)-\mathrm{F}(15) & 3.062 \\ \mathrm{~K}(5)-\mathrm{F}(16) & 3.078 \\ \mathrm{~K}(5)-\mathrm{F}(20) & 2.850 \\ \langle\mathrm{~K}(5)-\mathrm{F}\rangle & 2.892 \\ & \end{array}$

$\begin{array}{ll}\mathrm{Fe}(2)-\mathrm{F}(1 b) & 1.888 \\ \mathrm{Fe}(2)-\mathrm{F}(7 b) & 1.850 \\ \mathrm{Fe}(2)-\mathrm{F}(9) & 1.804 \\ \mathrm{Fe}(2)-\mathrm{F}(14) & 2.040 \\ \mathrm{Fe}(2)-\mathrm{F}(16) & 2.026 \\ \mathrm{Fe}(2)-\mathrm{F}(20) & 1.834 \\ \langle\mathrm{Fe}(2)-\mathrm{F}\rangle & 1.907 \\ & \\ \mathrm{~K}(2)-\mathrm{F}(1 b) & 2.539 \\ \mathrm{~K}(2)-\mathrm{F}(2) & 3.186 \\ \mathrm{~K}(2)-\mathrm{F}(6) & 2.736 \\ \mathrm{~K}(2)-\mathrm{F}(8 b) & 2.958 \\ \mathrm{~K}(2)-\mathrm{F}(10) & 2.668 \\ \mathrm{~K}(2)-\mathrm{F}(12) & 2.845 \\ \mathrm{~K}(2)-\mathrm{F}(13 c) & 3.092 \\ \mathrm{~K}(2)-\mathrm{F}(14) & 3.019 \\ \mathrm{~K}(2)-\mathrm{F}(18 c) & 2.648 \\ \mathrm{~K}(2)-\mathrm{F}(20) & 3.087 \\ \langle\mathrm{~K}(2)-\mathrm{F}\rangle & 2.878 \\ & \\ \mathrm{~K}(6)-\mathrm{F}(1 f) & 2.729 \\ \mathrm{~K}(6)-\mathrm{F}(2 f) & 2.762 \\ \mathrm{~K}(6)-\mathrm{F}(10) & 3.044 \\ \mathrm{~K}(6)-\mathrm{F}(13) & 2.677 \\ \mathrm{~K}(6)-\mathrm{F}(14) & 2.772 \\ \mathrm{~K}(6)-\mathrm{F}(16) & 3.024 \\ \mathrm{~K}(6)-\mathrm{F}(17) & 3.128 \\ \mathrm{~K}(6)-\mathrm{F}(18) & 3.031 \\ \mathrm{~K}(6)-\mathrm{F}(19) & 2.953 \\ \mathrm{~K}(6)-\mathrm{F}(20) & 2.883 \\ \langle\mathrm{~K}(6)-\mathrm{F}\rangle & 2.900 \\ & \\ & \\ & \\ & \\ & \\ & \end{array}$

$\mathrm{F}(3)-\mathrm{Fe}(1)-\mathrm{F}(18 a) \quad 177.1$

$\mathrm{F}(4)-\mathrm{Fe}(1)-\mathrm{F}(15 a) \quad 169.7$

$\mathrm{F}(5)-\mathrm{Fe}(1)-\mathrm{F}(16 a) \quad 178.6$

$\mathrm{F}(15 a)-\mathrm{Fe}(1)-\mathrm{F}(18 a) \quad 92.8$

$\mathrm{F}(16 a)-\mathrm{Fe}(1)-\mathrm{F}(18 a) \quad 88.4$

$\mathrm{F}(16 a)-\mathrm{Fe}(1)-\mathrm{F}(15 a) \quad 88.6$

$\mathrm{F}(3)-\mathrm{Fe}(1)-\mathrm{F}(15 a) \quad 87.2$

$\mathrm{F}(3)-\mathrm{Fe}(1)-\mathrm{F}(5) \quad 89.3$

$\mathrm{F}(4)-\mathrm{Fe}(1)-\mathrm{F}(5)$

$\mathrm{F}(1 b)-\mathrm{Fe}(2)-\mathrm{F}(16) \quad 172 \cdot 2$

$\mathrm{F}(7 b)-\mathrm{Fe}(2)-\mathrm{F}(20)$

$\mathrm{F}(9)-\mathrm{Fe}(2)-\mathrm{F}(14)$

$\mathrm{F}(1 b)-\mathrm{Fe}(2)-\mathrm{F}(9) \quad 97.7$

$\mathrm{F}(1 b)-\mathrm{Fe}(2)-\mathrm{F}(20)$

Symmetry code

\begin{tabular}{ll}
$\mathrm{Fe}(3)-\mathrm{F}(2)$ & 1.948 \\
$\mathrm{Fe}(3)-\mathrm{F}(6)$ & 1.906 \\
$\mathrm{Fe}(3)-\mathrm{F}(8 c)$ & 1.915 \\
$\mathrm{Fe}(3)-\mathrm{F}(13 c)$ & 1.903 \\
$\mathrm{Fe}(3)-\mathrm{F}(14 a)$ & 2.021 \\
$\mathrm{Fe}(3)-\mathrm{F}(17 a)$ & 1.998 \\
$\langle\mathrm{Fe}(3)-\mathrm{F}\rangle$ & 1.949 \\
& \\
$\mathrm{~K}(3)-\mathrm{F}(2)$ & 2.721 \\
$\mathrm{~K}(3)-\mathrm{F}(3 d)$ & 2.814 \\
$\mathrm{~K}(3)-\mathrm{F}(5 d)$ & 2.750 \\
$\mathrm{~K}(3)-\mathrm{F}(6)$ & 2.976 \\
$\mathrm{~K}(3)-\mathrm{F}(7)$ & 2.623 \\
$\mathrm{~K}(3)-\mathrm{F}(10)$ & 2.998 \\
$\mathrm{~K}(3)-\mathrm{F}(11)$ & 2.890 \\
$\mathrm{~K}(3)-\mathrm{F}(15 h)$ & 3.052 \\
$\mathrm{~K}(3)-\mathrm{F}(17 a)$ & 3.008 \\
$\mathrm{~K}(3)-\mathrm{F}(19 a)$ & 2.801 \\
$\langle\mathrm{~K}(3)-\mathrm{F}\rangle$ & 2.863 \\
& \\
$\mathrm{~K}(7)-\mathrm{F}(3 f)$ & 2.702 \\
$\mathrm{~K}(7)-\mathrm{F}(3 k)$ & 2.665 \\
$\mathrm{~K}(7)-\mathrm{F}(7 b)$ & 2.800 \\
$\mathrm{~K}(7)-\mathrm{F}(9)$ & 2.759 \\
$\mathrm{~K}(7)-\mathrm{F}(9 l)$ & 2.818 \\
$\mathrm{~K}(7)-\mathrm{F}(11 j)$ & 3.005 \\
$\mathrm{~K}(7)-\mathrm{F}(11 b)$ & 2.691 \\
$\mathrm{~K}(7)-\mathrm{F}(15)$ & 2.831 \\
$\mathrm{~K}(7)-\mathrm{F}(16)$ & 3.022 \\
$\mathrm{~K}(7)-\mathrm{F}(19 j)$ & 2.900 \\
$\langle\mathrm{~K}(7)-\mathrm{F}\rangle$ & 2.819 \\
& \\
\hline
\end{tabular}

$\mathrm{Fe}(4)-\mathrm{F}(10) \quad 1.828$

$\mathrm{Fe}(4)-\mathrm{F}(11) \quad 1.870$

$\mathrm{Fe}(4)-\mathrm{F}(12) \quad 1.943$

$\mathrm{Fe}(4)-\mathrm{F}(15 d) \quad 2.044$

$\mathrm{Fe}(4)-\mathrm{F}(17) \quad 2.045$

$\mathrm{Fe}(4)-\mathrm{F}(19) \quad 1.894$

$\langle\mathrm{Fe}(4) \cdot \mathrm{F}\rangle \quad 1.937$

$\mathrm{K}(4)-\mathrm{F}(1) \quad 3.042$

$\mathrm{K}(4)-\mathrm{F}(2) \quad 2.651$

$\mathrm{K}(4)-\mathrm{F}(4) \quad 2.656$

$\mathrm{K}(4)-\mathrm{F}(7) \quad 2.804$

$\mathrm{K}(4)-\mathrm{F}(8) \quad 2.740$

$\mathrm{K}(4)-\mathrm{F}(10) \quad 2.612$

$\mathrm{K}(4)-\mathrm{F}(13) \quad 2.657$

$\mathrm{K}(4) \cdot \mathrm{F}(14 i) \quad 3.215$

$\mathrm{K}(4) \mathrm{F}(20) \quad 2.697$

$\langle\mathrm{K}(4)-\mathrm{F}\rangle \quad 2.786$

$\mathrm{K}(8)-\mathrm{F}(1 b) \quad 2.989$

$\mathrm{K}(8)-\mathrm{F}(3) \quad 2.805$

$\mathrm{K}(8)-\mathrm{F}(4) \quad 2.591$

$\mathrm{K}(8)-\mathrm{F}(7 c) \quad 2.950$

$\mathrm{K}(8)-\mathrm{F}(9) \quad 3.015$

$\mathrm{K}(8)-\mathrm{F}(13 c) \quad 2.674$

$\mathrm{K}(8)-\mathrm{F}(16 a) \quad 3.045$

$\mathrm{K}(8)-\mathrm{F}(19 c) \quad 2.707$

$\begin{array}{ll}\langle\mathrm{K}(8)-\mathrm{F}\rangle & 2.847\end{array}$

$\mathrm{F}(11)-\mathrm{Fe}(4)-\mathrm{F}(17)$

$\mathrm{F}(12)-\mathrm{Fe}(4)-\mathrm{F}(19)$

$\mathrm{F}(15 d)-\mathrm{Fe}(4)-\mathrm{F}(10) \quad 177.0$

$\mathrm{F}(11)-\mathrm{Fe}(4)-\mathrm{F}(10)$

$\mathrm{F}(11)-\mathrm{Fe}(4)-\mathrm{F}(12)$

$\mathrm{F}(10)-\mathrm{Fe}(4)-\mathrm{F}(12) \quad 93.4$

$\mathrm{F}(15 d)-\mathrm{Fe}(4)-\mathrm{F}(17)$

$F(15 d)-F e(4)-F(19)$

$\mathrm{F}(17)-\mathrm{Fe}(4)-\mathrm{F}(19) \quad 84.7$

$\mathrm{Fe}(1 f)-\mathrm{F}(16)-\mathrm{Fe}(2) \quad 173.4$

$\mathrm{Fe}(2)-\mathrm{F}(14)-\mathrm{Fe}(3 f) \quad 162.0$

$\mathrm{Fe}(3 f)-\mathrm{F}(17)-\mathrm{Fe}(4) \quad 173.4$

$\mathrm{Fe}(1)-\mathrm{F}(15)-\mathrm{Fe}(4 f) \quad 172.8$ $\begin{array}{ll}\text { (a) } & x, y-1, z \\ \text { (d) } & x-\frac{1}{2}, y, \frac{1}{2}-z \\ \text { (g) } & \bar{x}, y+\frac{1}{2}, \bar{z} \\ \text { (j) } & \frac{1}{2}+x, y, \frac{1}{2}-z\end{array}$ (b) $\frac{1}{2}-x, \frac{1}{2}+x, z-\frac{1}{2}$

(e) $x-\frac{1}{2}, y+1, \frac{1}{2}-z$

(h) $\quad \frac{3}{2}-x, y+\frac{1}{2}, z-\frac{1}{2}$

(k) $\quad 1-x, \frac{1}{2}-z, z$ (c) $\frac{1}{2}-x, y-\frac{1}{2}, z-\frac{1}{2}$

(f) $x, 1+y, z$

(i) $\frac{1}{2}-x, y-\frac{1}{2}, \frac{1}{2}+z$

(l) $1-x, y+\frac{1}{2}, \bar{z}$ 
1179 independent reflexions were measured to a value of $2 \theta_{\max }=70^{\circ} .1088$ had $I>3 \sigma(I)$, where $\sigma(I)=$ [total counts + background $]^{1 / 2}$, and were considered to be observed. These intensities were corrected for Lorentz and polarization effects, but not for absorption $\left\{\mu[\lambda(\operatorname{Mo} K \alpha)]=55 \mathrm{~cm}^{-1}\right\}$.

\section{Determination and refinement of the structure}

The positions of the heavy atoms were determined from a Patterson synthesis. The $F$ atoms were located in a difference synthesis at an intermediate stage of refinement. The refinement was carried out in the noncentrosymmetric space group Pn2 ${ }_{1} a$ [4(a): $x, y, z$; $\left.\frac{1}{2}+x, y, \frac{1}{2}-z ; \frac{1}{2}-x, \frac{1}{2}+y, \frac{1}{2}+z ; \bar{x}, \frac{1}{2}+y, \bar{z}\right]$ which was indicated by a heavy concentration of vectors on the Harker plane $\left(u, \frac{1}{2}, w\right)$, as well as an acentric distribution of intensities. Full-matrix least-squares refinement (Busing, Martin \& Levy, 1962) with isotropic temperature factors reduced $R\left(=\Sigma|| F_{o}|-| F_{c} \| / \Sigma\left|F_{o}\right|\right)$ to
0.048 , based on a data-to-parameters ratio of $8 \cdot 5: 1$, with 128 independent parameters. A final $\left(F_{o}-F_{c}\right)$ synthesis confirmed the proposed solution.

$\sum w\left(\left|F_{o}\right|-\left|F_{c}\right|\right)^{2}$ was minimized with $w$ taken as unity for all reflexions. The overall scale factor was $1.305\left(F_{o}=k F_{c}\right)$. The form factors for $\mathrm{K}^{+}, \mathrm{Fe}^{3+}$ and $\mathrm{F}^{-}$were taken from McMaster, Kerr del Grande, Mallet \& Hubbel (1969), with the real and imaginary anomalous-dispersion terms given by Cromer (1965).

The final atomic and thermal parameters are given in Table 1. Table 2 contains the interatomic distances and angles. A table giving the calculated and observed structure amplitudes may be obtained on request from MV.*

* This table has also been deposited with the British Library Lending Division as Supplementary Publication No. SUP 32689 (8 pp.). Copies may be obtained through The Executive Secretary, International Union of Crystallography, 13 White Friars, Chester CH1 $1 \mathrm{NZ}$, England.

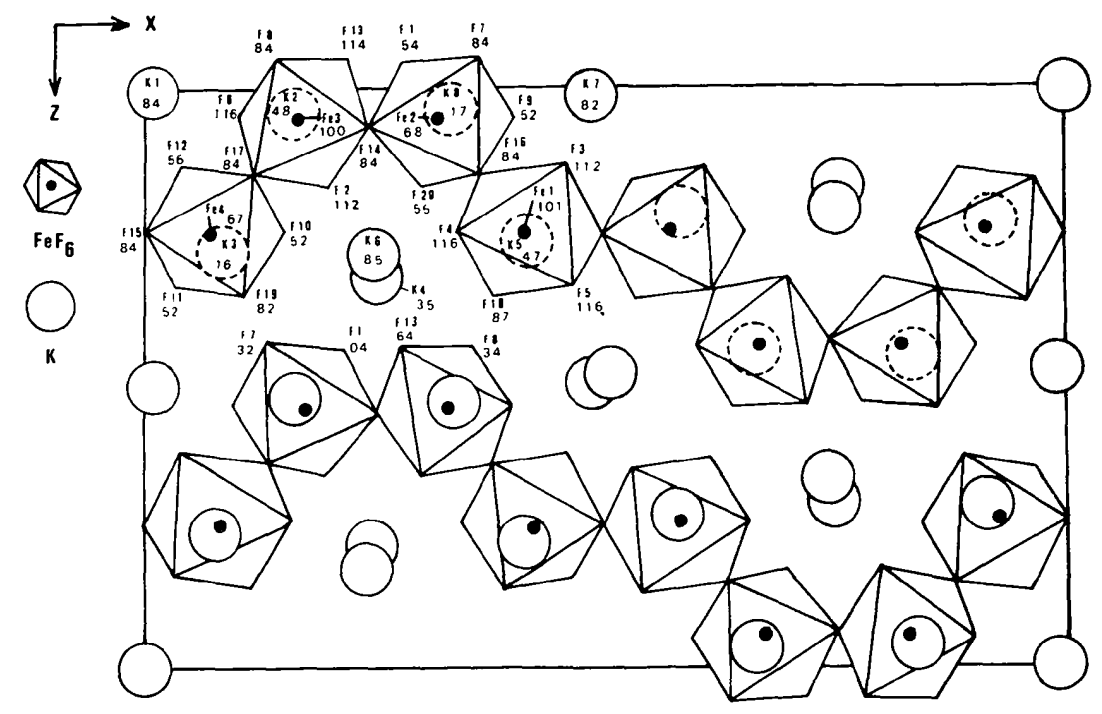

Fig. 1. Projection of the structure on the (010) plane.

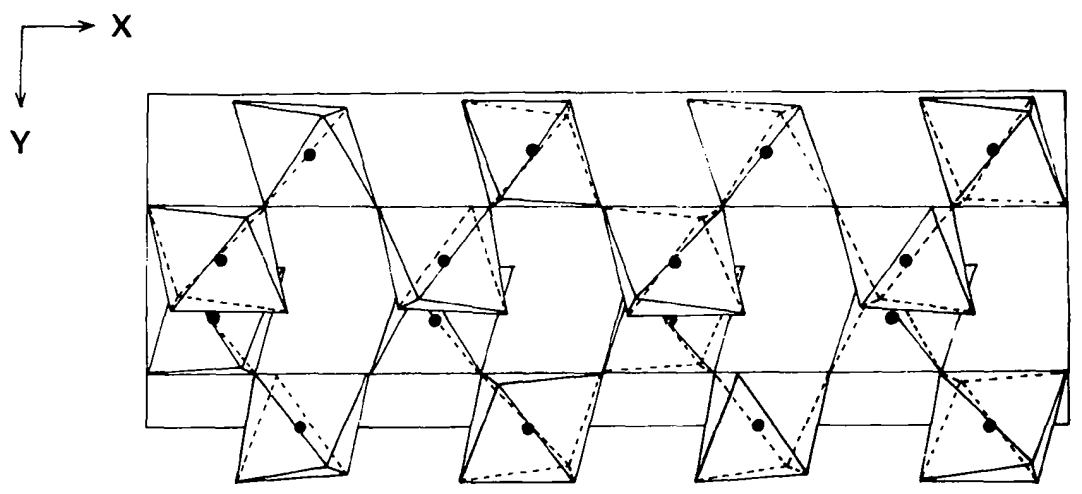

Fig. 2. Chains of $\mathrm{FeF}_{6}$ octahedra in $\mathrm{K}_{2} \mathrm{FeF}_{5}$. 


\section{Description and discussion of the structure}

Projections of the structure on (010) and (001) are shown in Figs. 1 and 2. The structure can be described as an assembly of infinite chains along [100] made up of octahedra sharing two of their corners. When two vertices of an octahedron are shared they can be either adjacent (cis) or opposite (trans). The sharing of two cis vertices leads to cyclic molecules or zigzag chains. On the other hand, a trans configuration leads to straight linear chains. In $\mathrm{K}_{2} \mathrm{FeF}_{5}$ we observe, in fact, zigzag cis chains of $\left[\mathrm{FeF}_{6}\right]_{n}^{2 n-}$. The $\mathrm{K}^{+}$ions with nineor tenfold coordination are found between the chains and assure their cohesion.

The $\mathrm{FeF}_{6}$ octahedra are regular with an average $\mathrm{Fe}-\mathrm{F}$ distance ranging from 1.907 to $1.949 \AA$ for four independent $\mathrm{Fe}$ atoms (Table 2). This is in agreement with the sum of their ionic radii, $1.925 \AA\left[r_{\mathrm{Fe}^{3}+}(\mathrm{VI})=\right.$ $0.645, r_{F-}$ (II) $=1.28 \AA$; Shannon \& Prewitt, 1969]. Similar average $\mathrm{Fe}-\mathrm{F}$ distances were observed in $\mathrm{CaFeF}_{5}(\mathrm{Fe}-\mathrm{F}=1.92 \AA$; von der Mühll \& Ravez, 1974) and in $\mathrm{SrFeF}_{5}(\mathrm{Fe}-\mathrm{F}=1.93 \AA$; von der Mühll, Daut \& Ravez, 1973). However, there seems to be a clear difference between the terminal and bridging $\mathrm{Fe}-\mathrm{F}$ bonds. The average $\mathrm{Fe}-\mathrm{F}_{t}, 1.882 \AA$, is clearly shorter than $\mathrm{Fe}-\mathrm{F}_{b}, 2 \cdot 020 \AA$. The same observation was made in $\mathrm{K}_{2} \mathrm{VO}_{2} \mathrm{~F}_{3}$ (Ryan, Martin \& Reisfeld, 1971) where $\mathrm{V}-\mathrm{F}_{t}=1.89 \AA$ and $\mathrm{V}-\mathrm{F}_{b}=2.10 \AA$, and in $\mathrm{RhF}_{5}$ (Morell, Zalkin, Tressaud \& Bartlett, 1973) where $\mathrm{Rh}-\mathrm{F}_{t}=1.808 \AA$ and $\mathrm{Rh}-\mathrm{F}_{b}=1.999 \AA$. This may be considered as an electrostatic effect, which is quite normal for a highly electronegative atom such as $\mathrm{F}$. The bridging $\mathrm{Fe}-\mathrm{F}-\mathrm{Fe}$ angles range from 162.0 to $173.4^{\circ}$ with an average of $170.4^{\circ}$. This angle is closer to the ideal angle of $180^{\circ}$ than the minimum of $132^{\circ}$. The average $\mathrm{K}-\mathrm{F}$ distances are in general shorter than the sum of the ionic radii of $1.91 \AA\left[r_{K+}(X)=1.58 \AA\right]$. This indicates the close-packed nature of the $\left[\mathrm{FeF}_{5}\right]_{n}^{2 n-}$ chains and $\mathrm{K}^{+}$ions in this structure.

The structures of a number of $A_{2} B \mathrm{~F}_{5}$-type compounds have been determined, such as $\mathrm{Tl}_{2} \mathrm{AlF}_{5}$ (Brosset, 1937), $\left(\mathrm{NH}_{4}\right)_{2} \mathrm{MnF}_{5}$ (Sears \& Hoard, 1969), $\mathrm{Rb}_{2} \mathrm{CrF}_{5}$ (Jacoboni, de Pape, Poulain, Le Marouille \& Grandjean, 1974) and $\mathrm{K}_{2} \mathrm{SmF}_{5}$ (Bochkova, Sav'yanov, Kuz'min \& Belov, 1973). $\mathrm{Tl}_{2} \mathrm{AlF}_{5}$ and $\left(\mathrm{NH}_{4}\right)_{2} \mathrm{MnF}_{5}$ are made up of linear octahedral trans chains held together by the $\mathrm{Tl}^{+}$and $\mathrm{NH}_{4}^{+}$ions in sixfold coordination. Both $\mathrm{Rb}_{2} \mathrm{CrF}_{5}$ and $\mathrm{K}_{2} \mathrm{FeF}_{5}$ possess similar octahedral zigzag cis chains with the cations in sixfold and nine- or tenfold coordination respectively.
The increase of the $A$ atom coordination and the shorter $A-\mathrm{F}$ distances in $\mathrm{K}_{2} \mathrm{FeF}_{5}$ compared with $\mathrm{Rb}_{2} \mathrm{CrF}_{5}$ imply a more compact structure, which leads us to believe that under pressure $\mathrm{Rb}_{2} \mathrm{CrF}_{5}$ will transform to a $\mathrm{K}_{2} \mathrm{FeF}_{5}$-type structure.

The octahedral chain character of this structure allows the prediction that $\mathrm{K}_{2} \mathrm{FeF}_{5}$ will show relatively one-dimensional magnetic behaviour with a low antiferromagnetic transition temperature. The magnetic study is presently under way.

\section{References}

Anderson, P. W. (1963). Magnetism, Vol. I, p. 25. London, New York: Academic Press.

Bochkova, R. I., Sav'yanov, Yu. N., Kuz'min, E. A. \& Belov, N. V. (1973). Dokl. Akad. Nauk SSSR, 212, 357-359.

Brosset, C. (1937). Z. anorg. allgem. Chem. 235, 139-147.

Busing, W. R., MARTIN, K. O. \& LeVY, H. A. (1962). ORFLS. Report ORNL-TM-305. Oak Ridge National Laboratory, Tennessec.

Cromer, D. T. (1965). Acta Cryst. 18, 17-23.

Goodenough, J. B. (1963). Magnetism and the Chemical Bond. New York: Interscience.

Jacoboni, C., De Pape, R., Poulain, M., Le Marouille, J. Y. \& Grandjean, D. (1974). Acta Cry'st. B30, 26882691.

McMaster, W. H., Kerr del Grande, N., Mallet, J. H. \& HubBel, J. H. (1969). Compilation of X-ray Cross Sections. Natl. Bur. Stand. UCRL-50174, Sec. II, Rev. 1.

Morell, B. K., Zalkin, A., Tressaud, A. \& Barti.ft, N. (1973). Inorg. Chem. 12, 2640-2644.

Mühll, R. VON DER, DAUT, F. \& RAVEZ, J. (1973). J. Solid State Chem. 8, 206-212.

Mühll, R. von DER \& Ravez, J. (1974). Rev. Chim. Minér. 11, 652-663.

Portier, J., Tressaud, A., De Pape, R. \& Hagenmuller, P. (1968). Mater. Res. Bull. 3, 433-436.

Ryan, R. R., Martin, S. H. \& Reisfeld, M. J. (1971). Acta Cryst. B27, 1270-1274.

Sears, D. R. \& Hoard, J. L. (1969). J. Chem. Phy's. 50, 1066-1072.

Shannon, R. D. \& Prewitt, C. T. (1969). Acta Cry'st. B25, 925-945.

Tressaud, A., Dance, J. M., Vlasse, M. \& Portier, J. (1976). C. R. Acad. Sci. Paris, Sér. C, 282, 1105-1108.

Tressaud, A., Portier, J., de Pape, R. \& Hagenmuller, P. (1970). J. Solid State Chem. 2, 269-277.

Vlasse. M., Menil. F.. Moriliere. C.. Dance, J. M., Tressaud, A. \& Portier, J. (1976). J. Solid State Chem. 17, 291-298.

W ANKLYN, B. M. (1975). J. Mater. Sci. 10, 1487-1493. 